\title{
DSS1/DSS2 astrometry for 1101 First Byurakan Survey blue stellar objects: Accurate positions and other results ${ }^{\star}$
}

\begin{abstract}
A. M. Mickaelian
Byurakan Astrophysical Observatory and Isaac Newton Institute of Chile, Armenian branch, Byurakan 378433, Aragatzotn province, Armenia

e-mail: aregmick@apaven.am

Received 22 January 2004 / Accepted 7 May 2004

Abstract. Accurate measurements of the positions of 1101 First Byurakan Survey (FBS) blue stellar objects (the Second part of the FBS) have been carried out on the DSS1 and DSS2 (red and blue images). To establish the accuracy of the DSS1 and DSS2, measurements have been made for 153 AGN for which absolute VLBI coordinates have been published. The rms errors are: $0.45^{\prime \prime}$ for DSS1, $0.33^{\prime \prime}$ for DSS2 red, and 0.59" for DSS2 blue in each coordinate, the corresponding total positional errors being $0.64^{\prime \prime}, 0.46^{\prime \prime}$, and $0.83^{\prime \prime}$, respectively. The highest accuracy $\left(0.42^{\prime \prime}\right)$ is obtained by weighted averaging of the DSS1 and DSS2 red positions. It is shown that by using all three DSS images accidental errors can be significantly reduced. The comparison of DSS2 and DSS1 images made it possible to reveal positional differences and proper motions for 78 objects (for 62 of these for the first time), including new high-probability candidate white dwarfs, and to find objects showing strong variability, i.e. high-probability candidate cataclysmic variables.
\end{abstract}

Key words. astrometry - stars: novae, cataclysmic variables

\section{Introduction}

The Digitized Sky Survey (DSS) is a project of the STScI to cover the entire sky in several colors. Its primary goal was to produce the Hubble Guide Star Catalog (GSC). The plates of the Palomar Observatory Sky Surveys (POSS) and UKST SERC surveys were digitized using a modified PDS microdensitometer. Each plate produced by the DSS covers $6.5^{\circ} \times$ $6.5^{\circ}$ of the sky. Two surveys are available so far: DSS1 (Lasker et al. 1990) and DSS2 (Lasker et al. 1996). DSS1 is a source for the identification of various kinds of objects and has also been used by a number of authors for accurate coordinate measurements (Schneider et al. 1992; Bowen et al. 1994; Kirhakos et al. 1994; Véron-Cetty \& Véron 1996; Bicay et al. 2000). There is a significant improvement of the limiting magnitudes and spatial resolution in DSS2 compared to DSS1, which is especially useful for understanding the structure of extended objects, as well as for resolving binary images. On the other hand, DSS2 can be used together with DSS1 to search for proper motions (PM), variable objects, etc. Finally, DSS2 can be used for hopefully better astrometric measurements (due to its better astrometric solution). In this paper, using absolute VLBI positions for 153 AGN, we first tried to determine the accuracy of the measurements from DSS1 and compare it with that of

* Table 1 is only available in electronic form at the CDS via anonymous ftp to cdsarc.u-strasbg.fr $(130.79 .128 .5)$ or via http://cdsweb.u-strasbg.fr/cgi-bin/qcat?]/A+A/426/367 previously published papers and, for the first time, for DSS2. Then we made accurate measurements for 1101 FBS blue stellar objects. Having carried out a cross-correlation with the Minnesota Automated Plate Scanner (MAPS) catalog (Cabanela et al. 2003), we compared our results with its positions too. We show that careful study and comparison of DSS1 and DSS2 images can reveal compact galaxies (candidate $\mathrm{AGN}$ ), proper motion, and variable stars.

\section{The FBS blue stellar objects}

The FBS is the largest spectral survey in the Northern sky. It was carried out by Markarian, Lipovetski and Stepanian in 1965-1980 with the Byurakan Observatory $1 \mathrm{~m}\left(40^{\prime \prime}\right)$ Schmidt telescope using a $1.5^{\circ}$ prism (Markarian 1967). The FBS covers an area of $17000 \mathrm{deg}^{2}$ defined by $\delta>-15^{\circ}$ and $|b|>15^{\circ}$. The limiting magnitude is $17.5^{\mathrm{m}}-18^{\mathrm{m}}$, the dispersion is $1800 \AA / \mathrm{mm}$ near $\mathrm{H} \gamma$. The FBS was conducted originally to search for galaxies with UV-excess (UVX). 1515 UVX galaxies have been discovered (Mazzarella \& Balzano 1986; Markarian et al. 1989). The Second part of the FBS was devoted to the discovery and study of blue (UVX) stellar objects (Abrahamian \& Mickaelian 1996, and references therein). It was carried out in 278 fields, in a $4009 \mathrm{deg}^{2}$ area defined by $+33^{\circ}<\delta<+45^{\circ}$ and $\delta>+61^{\circ}$ ). The discovery of new bright QSOs, Seyferts, planetary nebula nuclei, cataclysmic variables (CV), white dwarfs (WD), subdwarfs, and other interesting objects was the main purpose of this work. 
It is similar to the Palomar-Green (PG) survey (Green et al. 1986) but uses a spectroscopic method of selection (which is more efficient), has a deeper magnitude limit, and larger area (though the Second part has not yet covered the whole FBS area so far). 1103 blue stellar objects (BSOs) have been selected, including 716 new ones. They have been published in 11 lists. The catalog of the FBS BSOs is available at the CDS (Abrahamian et al. 1999).

The Second part of the FBS has proved to be a source of QSOs, Seyferts, WDs, CVs, etc. (Mickaelian et al. 1999, 2001, 2002). Altogether, spectroscopic observations have been carried out for $\sim 400$ FBS objects; however, only 100 have been published so far. Based on new spectroscopic observations, the local density of QSOs and the completeness of the Bright Quasar Survey (BQS) (Schmidt \& Green 1983) have been re-estimated. For a review of the FBS blue stellar objects see Mickaelian (2000).

The FBS objects were published before the DSS was available, and the coordinates were measured from the POSS paper prints with an accuracy of less than $1^{\prime}$ for the first 4 lists, and of $\sim 0.1^{\prime}$ for the next 7 lists. Finding charts were published only for the first 4 lists, and in fact, it is impossible to find the objects from the next lists. For further study of the FBS objects we find it useful to give accurate optical positions measured from the DSS. Moreover, we have measured positions from both DSS1 and DSS2 (red and blue), compared the results, and estimated their accuracy.

\section{Measurements}

\subsection{The DSS1 and DSS2 and their astrometric solutions}

The First Digitized Sky Survey (DSS1) (Lasker et al. 1990) is the digitized database of 2 surveys: the Palomar Observatory Sky Survey 1 (POSS1), carried out in 1948-1958 with the Palomar Oschin $1.2 \mathrm{~m}$ Schmidt telescope, and the SERC J Southern Sky Survey, carried out with the UKST $1.2 \mathrm{~m}$ Schmidt telescope in 1975-1987. The Second Digitized Sky Survey (DSS2) (McLean 2000) is the digitized database of two new surveys (POSS2 and AAO-SES) carried out with the same telescopes in 1987-2000. DSS2 has been completed in 3 colors (IIIaJ, IIIaF and IV-N), the first two being approximately similar to DSS1 blue $(103 \mathrm{aO}$, being currently digitized) and red (103aE), and have limiting magnitudes of $22.5^{\mathrm{m}}$ and $20.8^{\mathrm{m}}$, respectively, compared to $21.0^{\mathrm{m}}$ and $20.0^{\mathrm{m}}$ for the DSS1. Thus, DSS1 red (hereafter DSS1), DSS2 red (hereafter DSS2r), and DSS2 blue (hereafter DSS2b) are available. In DSS2, the digitization has been made with a smaller sampling ( $15 \mu$ or $1^{\prime \prime} /$ pix compared to $25 \mu$ or $1.67^{\prime \prime} /$ pix for DSS 1 ). There is a significant improvement both in magnitude limits (hence, good images for fainter objects), and resolution.

The astrometric reference catalogs used for the DSS1 are AGK3 (Heckman et al. 1975) for $\delta>2^{\circ}$, the SAOC (SAO 1966) for $-65^{\circ}<\delta<2^{\circ}$ and the CPC (Stoy 1968) for $\delta<-65^{\circ}$ (Russell et al. 1990). As mentioned by Véron-Cetty \& Véron (1996), the astrometric solution and the accuracy at the plate edges may be worse, but is not systematic; it depends on the distribution of the reference stars between the object and the plate edge. According to McLean (2000), the DSS2 astrometric (as well as photometric) calibration is significantly better than that of the DSS1, due to the use of new astrometric standards from the Second Generation GSC (Lasker et al. 1998), the Tycho-2 catalog (Hog et al. 2000) with astrometric standard errors of $0.06^{\prime \prime}$, the ICRS reference frame, and the improvement in measurement precision. Classical polynomial astrometric solutions have been made for both surveys. However, only a large number of measurements is able to establish the true astrometric accuracy for both surveys.

\subsection{AGN with accurate VLBI positions}

The DSS1 makes it possible to obtain an accuracy of 1" or better (Véron-Cetty \& Véron 1996; Bicay et al. 2000). For the first time we have carried out measurements for the same objects with DSS1, DSS2r and DSS2b. For the DSS2, an improvement in accuracy can be expected. However, we can test the accuracy of the astrometric solution only by measuring a significant number of objects. For this, we have repeated measurements for the sample of 153 AGN compiled by Véron-Cetty \& Véron (1996) for which there are published absolute VLBI positions with rms errors of 2 mas (Sovers et al. 1988; Ma et al. 1990; Robertson et al. 1993). The software FITSView v.2.0.2 (NRAO; Cotton 1999) has been used. It makes it possible to select and display images, mark the positions of objects and measure the coordinates and pixel values of the image. Two kinds of measurement are available: a manual cursor sampling, and a 2D Gaussian-fitting method to find the center of the image.

First, we have carried out measurements for all $153 \mathrm{ob}-$ jects on DSS1 and DSS2r. For DSS2b, only 79 images were available. A number of objects has positional differences larger than $1^{\prime \prime}: 39$ from DSS1, 23 from DSS2r and 26 from DSS2b, including a few with differences larger than $2^{\prime \prime}$; the largest errors being 4.5" for DSS1, 3.0" for DSS2r, and 3.6" for DSS2b. This proves that DSS2r gives a significant advantage in accuracy. DSS2b measurements are probably not so good because of saturation for many images, AGN being blue. Checking all images for objects having $>1^{\prime \prime}$ differences shows that the errors occur for the following reasons: extended images; binary or very close (blended) images; elongated images (plate defects); very faint or bad (asymmetric) images (mostly in DSS1); very bright (saturated) images; objects near the plate edges (radial effect according to Véron \& Hawkins 1995).

In all, we find 45 objects having explainable relatively poor accuracy, including those that are extended (4), have faint (28) or asymmetric (11) images, or are saturated (2), objects with binary images or near the plate limit having been excluded before by Véron-Cetty \& Véron (1996). For a number of faint and saturated images, we have re-measured positions with the cursor instead of the Gaussian. This improves the accuracy but is still worse than the Gaussian measurements for normally exposed objects (cursor measurements have an additional error of up to 0.5 pix, i.e. $0.8^{\prime \prime}$ for DSS1 and $0.5^{\prime \prime}$ for DSS2). Therefore, to estimate the true accuracy we have excluded all 45 abovementioned objects. In fact we have applied the same criteria 
to avoid inaccurate measurements as Véron-Cetty \& Véron (1996) (near the plate limit or blended images) with somewhat stronger restrictions.

One object (S4 0707+476) is a special case. For this object, all measurements give a systematic shift from its VLBI radio position of $3.0^{\prime \prime}-3.6^{\prime \prime}$ to NE. According to Stickel \& Kuehr (1994), the QSO with $z=1.292$ has an optical magnitude $18.2^{\mathrm{m}}$, so we measure a bright star which is different (MAPS O magnitude $14.46^{\mathrm{m}}$ ). According to Meisenheimer \& Roser (1983), it is a superposition of a bright F8V galactic star and an extended faint object (AGN). Thus, we should exclude this object from the measurements.

Out of 107 objects, now only 7 from the DSS1, 4 from DSS2r, and 12 from DSS2b (for 52 objects) have positional differences larger than $1^{\prime \prime}$ (the largest having 4.5", 1.8", and $2.2^{\prime \prime}$ respectively).

The DW $0202+31$ positional error of $4.5^{\prime \prime}$ (in Dec) cannot be explained by the above-mentioned reasons. The object is not very near the plate edge $(|x|=14.7,|y|=8.1, x$ and $y$ being the distance from the $36 \mathrm{~cm} \times 36 \mathrm{~cm}$ size plate center). For this object, Véron-Cetty \& Véron (1996) give 5.4" difference with VLBI from one plate (which has the object farther from the plate edge), and $0.2^{\prime \prime}$ for the other plate. The radial distance from the center is $16.8 \mathrm{~cm}$ and $17.5 \mathrm{~cm}$ for these plates, respectively. Thus, the accidental error comes from one DSS1 plate (its astrometric solution is probably inaccurate), which has been corrected both by Véron-Cetty \& Véron (1996), and by our checking in DSS2r and DSS2b. Thus, the best measurement is not always given by the plate having the object nearest to its center. This is an example of how we can avoid errors by several measurements.

Figure 1 shows the positional differences between our positions and the absolute VLBI positions for 153 AGN measured in DSS1 (upper left panel) and DSS2r (upper right panel), and the same after exclusion of objects with extended, saturated and faint images (lower panels), for 107 objects. In both we find an improvement of accuracy from DSS1 to DSS2. Without correction for plate edge effects, we obtain $0.45^{\prime \prime}$ rms accuracy for DSS1 (excluding the object with accidental error of $4.5^{\prime \prime}$ ), $0.33^{\prime \prime}$ for DSS2r, and $0.59^{\prime \prime}$ for DSS2b in each coordinate. An improvement from $0.6^{\prime \prime}$ to $0.45^{\prime \prime}$ has been made compared with the accuracy obtained by Véron-Cetty \& Véron (1996) for DSS1 due to the exclusion of objects with measurement problems. The corresponding values for the total positional errors are $0.64^{\prime \prime}, 0.46^{\prime \prime}$ and $0.83^{\prime \prime}$, respectively.

We find that DSS2b does not always give good positions, probably because of poor astrometric solution. The internal agreement between our 3 measurements is $0.66^{\prime \prime} \mathrm{rms}$ between DSS1 and DSS2r, and 0.77" rms between DSS2r and DSS2b. We have checked the DSS1 and DSS2r images in case of large differences. In addition to DW $0202+31$, we find different astrometric solutions between DSS1 and DSS2r in $7(4.6 \%)$ cases (all objects in the field have systematic differences between DSS1 and DSS2r). All of them have errors larger than $1^{\prime \prime}$ but cannot be explained by any of the abovementioned reasons. We have checked these objects with VLBI to find out which solution is better: for 2 objects, 4C 47.08 and S5 0836+71, errors come from DSS2r (1.8" and 1.0",
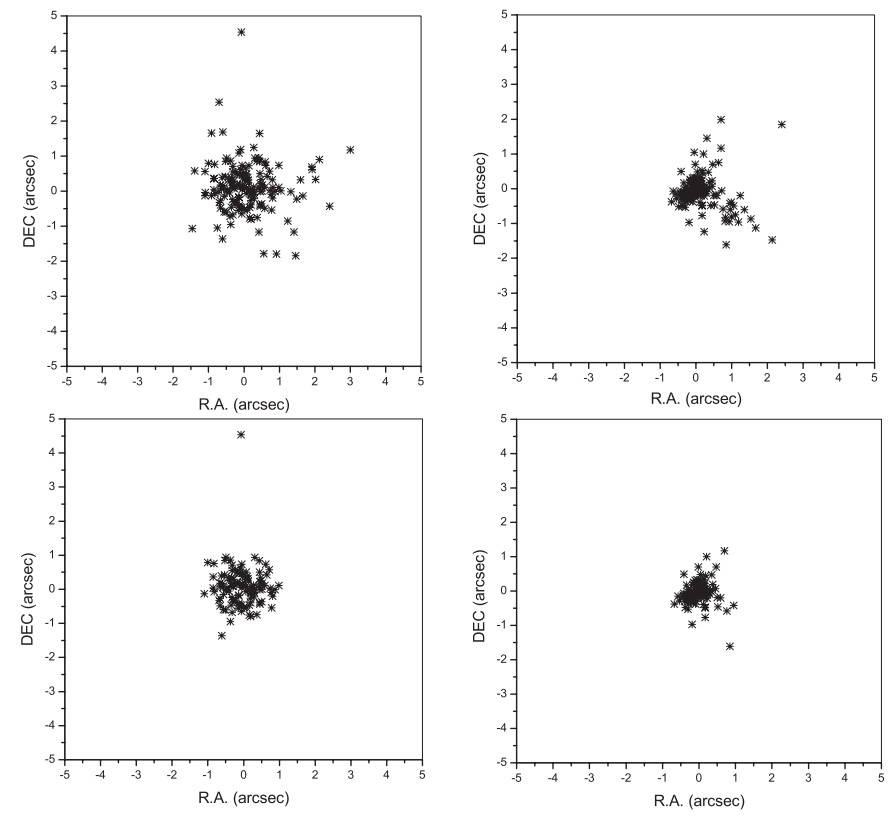

Fig. 1. DSS1 (left) and DSS2r (right) vs. VLBI positional differences for 153 AGN (upper panels), and for 107 AGN after exclusion of objects with poor measurements (lower panels).

respectively), and for 5 others (PKS 0332-403, 0402-362, 0420-01, 0723-008 and 1045-18, all in the South), the errors $\left(1.1^{\prime \prime}-1.8^{\prime \prime}\right)$ ) are in DSS1 (excluding the large difference of $\left.4.5^{\prime \prime}\right)$. We conclude that the best measurements are based on DSS2r, though, to avoid accidental errors, it is better also to check the DSS1 positions. If averaged with corresponding weights (DSS1 $1^{2}: D S S 2 r^{2}=1: 1.94$ for 106 objects, excluding the one with $4.5^{\prime \prime}$ error), DSS1 and DSS2r together give $0.42^{\prime \prime}$ rms compared to the VLBI positions $\left(0.30^{\prime \prime}\right.$ in each coordinate). Only 3 objects have errors $>1.0^{\prime \prime}$ (one $1.1^{\prime \prime}$, and two $\left.1.2^{\prime \prime}\right)$. Thus we avoid accidental errors and make a slight improvement in rms, reaching the maximum available accuracy. More importantly, we are left with good positions for almost all objects.

\subsection{The plate edge effect}

Surprisingly, the three objects having $>1^{\prime \prime}$, are not near the plate edges (all are within $10 \mathrm{~cm}$ in both $x$ and $y$ axes). To check if there are plate edge effects (increases of the positional error near the plate edges), we built up a synthetic (fake) plate for 107 AGN having absolute VLBI positions. Figure 2 shows that there is in fact no significant plate edge effect. Moreover, all objects having $>1$ " errors in DSS 1 have a smaller error in DSS2r, and vice versa. The largest error in DSS2r $\left(1.8^{\prime \prime}\right.$ in Dec) is near the plate center. In Fig. 3 we show the change of errors in RA with respect to the plate $\mathrm{x}$-axis, and Dec with respect to the plate $y$-axis for DSS1 and DSS2r separately. Both are negligible.

To check for a radial effect, we plot the positional errors with respect to $r$ (distance from the plate center), and average errors in intervals of $2 \mathrm{~cm}$ from the plate center both for DSS1 

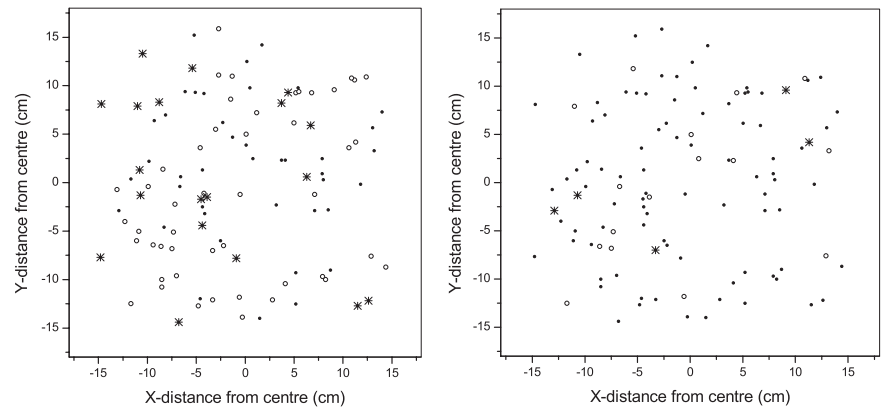

Fig. 2. Distribution of 107 AGN on a synthetic POSS plate with their positions relative to the plate center (for DSS1 on the left panel and DSS2r on the right panel). Positional errors within $0.5^{\prime \prime}$ are shown as dots, errors of $0.51^{\prime \prime}-1.00^{\prime \prime}$ as open circles and errors larger $1.0^{\prime \prime}$ as asterisks. The objects are distributed randomly showing that there is no significant plate edge effect.
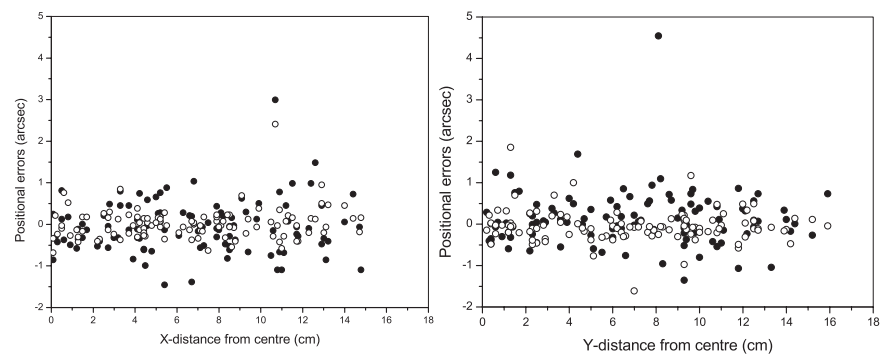

Fig. 3. Distribution of positional errors with respect to $x$ - (left panel) and $y$ - (right panel) distances from the plate centre. Black circles are for DSS1 and open circles are for DSS2r. There is no systematic change eighter along $x$ or $y$.
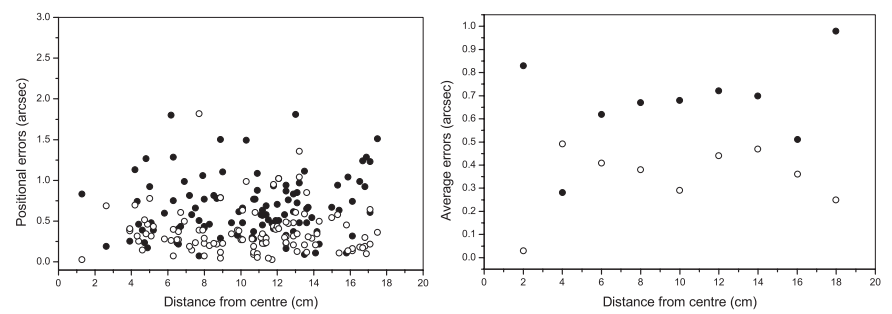

Fig. 4. Distribution of individual (left panel) and average (right panel) positional errors with respect to distance from the plate centre. Black circles are for DSS1 and open circles are for DSS2r.

and DSS2r (Fig. 4). Note that there is only one object with $r<$ $2 \mathrm{~cm}$, and three with $2 \mathrm{~cm}<r<4 \mathrm{~cm}$, so the first 4 points are not very certain. For the other intervals, we have $8-25$ objects in each. We do not see a difference with $r$ : DSS1 errors are typically within $0.6^{\prime \prime}-0.7^{\prime \prime}$, and DSS2r errors within $0.4^{\prime \prime}-0.5^{\prime \prime}$.

Finally, Fig. 5 is the same as in Véron-Cetty \& Véron (1996): the projection of the vector $(\Delta \alpha, \Delta \delta)$ on the radiusvector vs. distance from the plate center. We see that there is no significant plate edge effect for either DSS1 or DSS2. This effect (described by Véron \& Hawkins 1995) may play a role only for objects situated at the very edges of the POSS plates $(r>18 \mathrm{~cm})$, and for those regions where there were not enough reference stars for the astrometric solution. We conclude that taking into account the plate solution procedure (especially for DSS2r) this effect should be negligible. We have decided

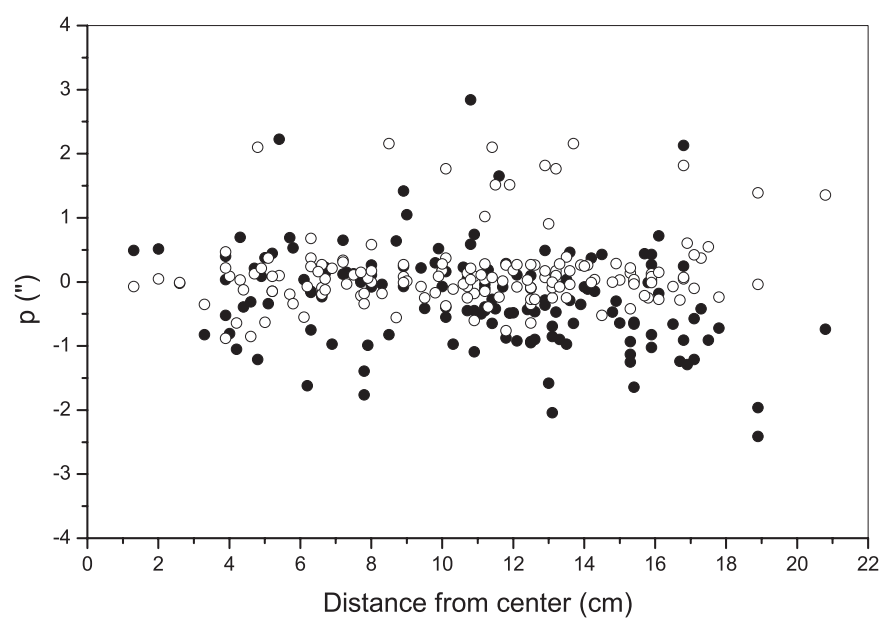

Fig. 5. Plot of the projection of the error vector $(\Delta \alpha, \Delta \delta)$ on the radius vector of the plate vs. distance from the plate center for 107 AGN. Black circles are for DSS1 and open circles are for DSS2r. A slight deviation can be noticed near the plate edge for DSS1; however, there is no change for the DSS2r measurements.

to measure the positions of the FBS blue stellar objects in all 3 DSS images (without correction for plate edge effect), to obtain the best available accuracy and to follow differences between 2 epochs, as well as between images in 2 colors.

\subsection{FBS blue stellar objects}

Accurate DSS1 positions have been published for 195 FBS objects so far (Mickaelian et al. 1999), however, we have repeated the measurements using the DSS2 as well. The finding charts for objects FBS \# 1-429 were published in the original papers, except for objects having previously published finding charts (mostly from the PG catalog). No charts were available for other objects. A number of extremely poor positions and misprints has been found in the FBS catalog as available at the CDS, both coming from the original papers and occurring later. All these errors have been checked and corrected.

For one object there was a misprint of the name in the catalog: FBS $1715+409$ should be FBS $1715+406$. The published finding charts for FBS 212 and FBS 213 are the same, but the objects are different. The name of FBS 336 has a misprint: it should be FBS $1413+420$ A rather than $1313+420 \mathrm{~A}$, and FBS 538 should be FBS $1323+333$ rather than $1223+333$. Moreover, two different objects have been listed in the cata$\log$ as FBS $336(1413+420 \mathrm{~A})$ and FBS $337(1413+420 \mathrm{~B})$ with the same finding chart (one in the PG catalog, one in FBS). We have checked the original data and concluded that these objects are the same. FBS 1038 and 1099, found from different plates at different times, are also identical. Thus, in fact, the FBS catalog contains 1101 objects rather than 1103 .

Some misprints and large errors in the FBS catalog happened during the survey or came from the PG catalog for the associated objects. Ten objects had misprints in their published positions in the FBS lists: FBS 34 (30' in RA), FBS $52\left(30^{\prime}\right.$ in Dec), FBS 109 (10' in Dec), FBS 137 (10' in Dec), FBS 153 $\left(20^{\mathrm{s}}\right.$ in RA), FBS 202 ( $2^{\mathrm{m}}$ in RA), FBS 222 (10' in Dec), 
FBS $425\left(1^{\mathrm{m}}\right.$ in RA), FBS 770 (misprint in Dec arcminutes 37 instead of 31$)$ and FBS 1002 ( $1^{\circ}$ in Dec). Other objects, all from the first lists, had just very poor positions (a few arcmin). 4 misprints have been found in PG: PG $0836+619$ $\left(20^{\mathrm{s}}\right.$ in RA), PG $1047+694\left(10^{\mathrm{s}}\right.$ in RA), PG $1606+627\left(15^{\mathrm{s}}\right.$ in RA), PG $1620+648\left(20^{\mathrm{s}}\right.$ in RA). All these misprints have been corrected.

Inaccurate measurements for saturated and faint images, etc. occurred for the FBS objects, as for AGN having VLBI positions. For 44 objects we had to measure positions with the cursor (giving the central pixel as seen by eye) (marked in Table 1), these positions have an additional inaccuracy of $0.8^{\prime \prime}$ for DSS1, and $0.5^{\prime \prime}$ for DSS2. For a number of saturated bright stars (mostly in DSS2), the images are not round, and the Gaussian does not work properly. As a result, the worst positions we have are in DSS2b (because the objects are blue and are brighter in DSS2b), in addition to a poor astrometric solution for DSS2b found by the AGN with VLBI positions. For close binary images all measurements have been done manually (by cursor). Working with 3 images allowed us to control all inaccuracies and accidental errors in the measurements.

\section{Results}

\subsection{DSS1/DSS2 positional differences}

1101 FBS objects have been measured in all 3 images: DSS1, DSS2r and DSS2b (two objects, FBS 1096 and FBS 1098, are absent in all 3 images, though they are very bright in the FBS; their coordinates have been measured approximately using the FBS position and the nearby stars). First we estimate the differences between these measurements. We should not forget, however, that there are WDs and subdwarfs among the FBS objects, which may have proper motions (PM), and some differences between DSS1 and DSS2 (and sometimes, between DSS2r and DSS2b) may be due to this. Hence it is difficult to estimate the real differences between DSS2 and DSS1. However, $52 \%$ of the differences are $<1^{\prime \prime}, 68 \%<$ $1.5^{\prime \prime}$ and $79 \%<2$, the largest difference being 23.8". The rms between the two measurements is $2.37^{\prime \prime}$, which is much larger than that for the VLBI sample. 42 objects had differences $\geq 2.5^{\prime \prime}$ between the DSS2r and DSS2b positions (the largest being $3.9^{\prime \prime}$ ), and the rms is $0.98^{\prime \prime}$. We have checked the images of 42 objects that had large differences and found that all are very bright and saturated. We re-measured them centering the cursor manually and got rid of all differences $\geq 2.5^{\prime \prime}$. Two objects, FBS 197 and FBS 574 have large PM and large differences between the epochs of the DSS2r and DSS2b plates so that the differences are due to PM (see subsection Proper motions). Still, only $68 \%$ of the objects have $<1$ " accuracy if compared from DSS2r and DSS2b. Figure 6 is the plot of the differences between DSS2r and DSS1 (left panel), and DSS2r and DSS2b (right panel) measurements respectively. While the DSS2r-DSS2b position difference distribution is similar to that for VLBI objects, we find a significant increase in DSS2r-DSS1 differences. We conclude that a number of objects has large PM.
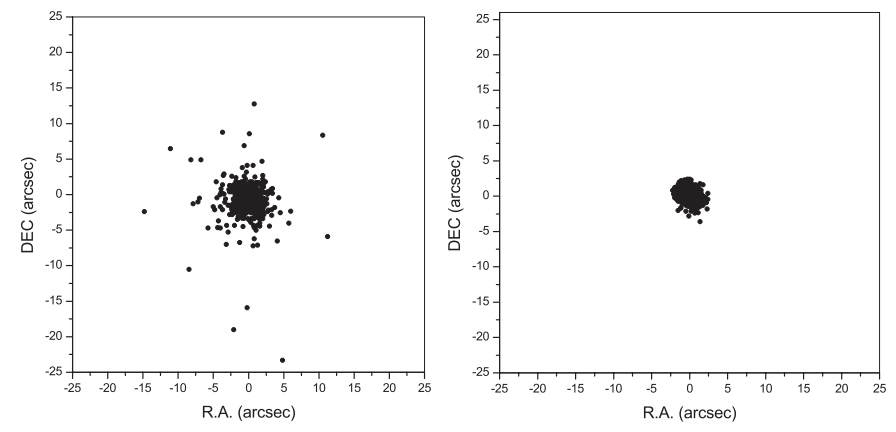

Fig. 6. DSS2r-DSS1 (left panel) and DSS2r-DSS2b (right panel) positional differences for 1101 FBS BSOs. Large differences in the left panel are due to PM.
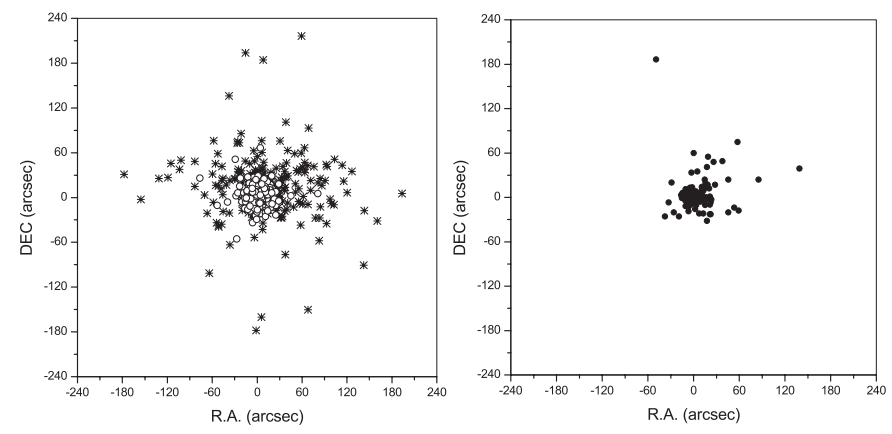

Fig. 7. Plot of the differences between the measured positions and the previously available positions from FBS (left panel) and PG (right panel) measurements. Objects of lists 1-4 (asterisks) and 5-11 (open circles) are shown separately. There is a significant improvement of positional accuracy for measurements from both catalogues.

\subsection{Accuracy of the previous positions}

We have compared the results obtained by accurate measurements with the DSS and previously published data. When measuring from the POSS charts, we had made a significant improvement in measurements starting with the 5th list. So we discuss objects from lists 1-4 and lists 5-11 separately. The comparison is made of course with DSS1 measurements to avoid differences due to PM, as the FBS original positions had been estimated from POSS1. We have excluded (corrected) misprints and large accidental errors for 10 objects (described above). The rms is $35^{\prime \prime} .8$, the largest error (for FBS 99) being $3^{\prime} 44^{\prime \prime}$. Figure 7 shows the differences between our new positions and the original ones for 834 FBS objects (leaving out PG objects which had coordinates from the PG catalog), and the same for 265 PG objects, showing some accidental errors in the original PG positions (excluding the 4 misprints mentioned above). Indeed, according to Bicay et al. (2000), a number of PG objects had poor positions (10" and larger). For the 265 PG objects studied, the rms is 16 "' 8 . In the left panel of Fig. 7, objects from lists 1-4 (317 objects) and 5-11 (517) are shown separately. A significant improvement from 59!'2 rms to 14 ". 9 rms was made at that time (1990) by using new POSS grids (and a new method for coordinate measurements). 


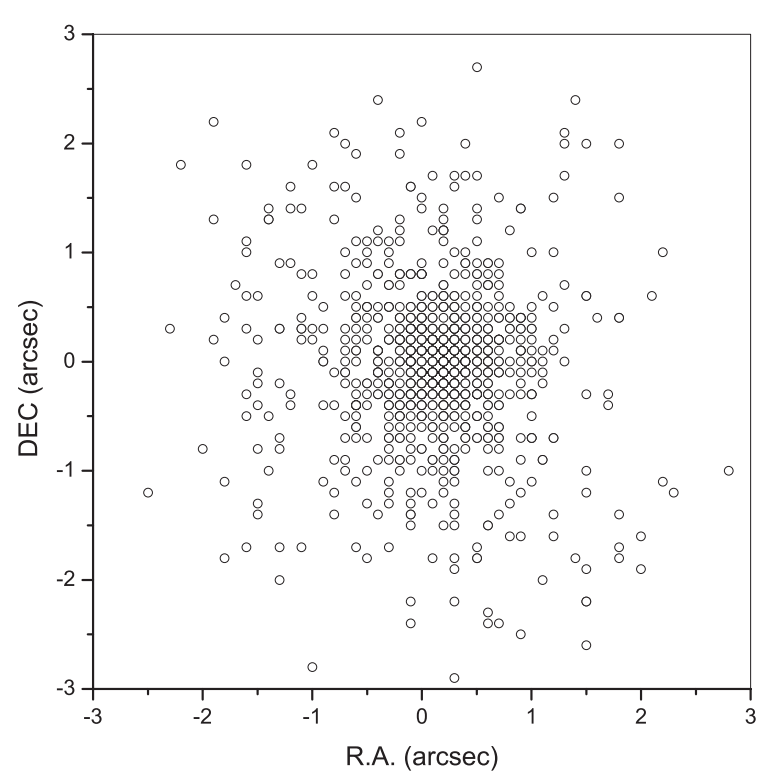

Fig. 8. MAPS-FBS positional differences for all 913 FBS BSOs found in MAPS.

\subsection{The list of FBS objects}

The new list of FBS objects with accurate positions as measured from DSS1 and DSS2r images is given in Table 1. The consecutive columns list: 1 - FBS \#; 2 - FBS name (B1950 hhmm+ddd); 3, 4 - J2000 optical position measured from DSS1 with an rms accuracy of 0.45"; 5 - "C" denotes cursor measurements (instead of Gaussian fit) corresponding to $\sim 1.5^{\prime \prime}$ accuracy; $6,7-\mathrm{J} 2000$ optical position measured from DSS2r with an rms accuracy of $0.33^{\prime \prime} ; 8-{ }^{-} \mathrm{C}$ " denotes cursor measurements with an accuracy of $\sim 1^{\prime \prime} ; 9$ - differences between the DSS2r and DSS1 positions; 10 - differences between the DSS2r and DSS2b positions, and 11 - comments. The position corresponding to each epoch is given to help users interested in PM stars.

\subsection{MAPS coordinates}

Having accurate positions for the FBS objects allowed us crosscorrelate the FBS list with the MAPS Catalog (Cabanela et al. 2003). A 3" radius has been used, assuming that our accuracies and those of MAPS will make it possible to find all objects in the area (FBS goes down to $|b|=15^{\circ}$, and sometimes even to $12^{\circ}$, and not all objects are in MAPS). The DSS1 positions have been given, as large PM would not make it possible find many objects with DSS2 positions. For 1103 objects in the list, 1083 coincidences have been found corresponding to 913 objects, including 752 found once, 152 found twice, and 9 found thrice; 190 were not found. For all objects found more than once, average positions, magnitudes and colors have been taken. In case of three values, when two of them were very close and the third was different ( 28 out of 36 cases), the average of the two closest values has been taken, the third being believed to be erroneous. The MAPS classification into "s" and " $\mathrm{g}$ " has also been revised. When different for the same object ( 6 cases out of 161, FBS 102, 372, 676, 738, 743, and 762,

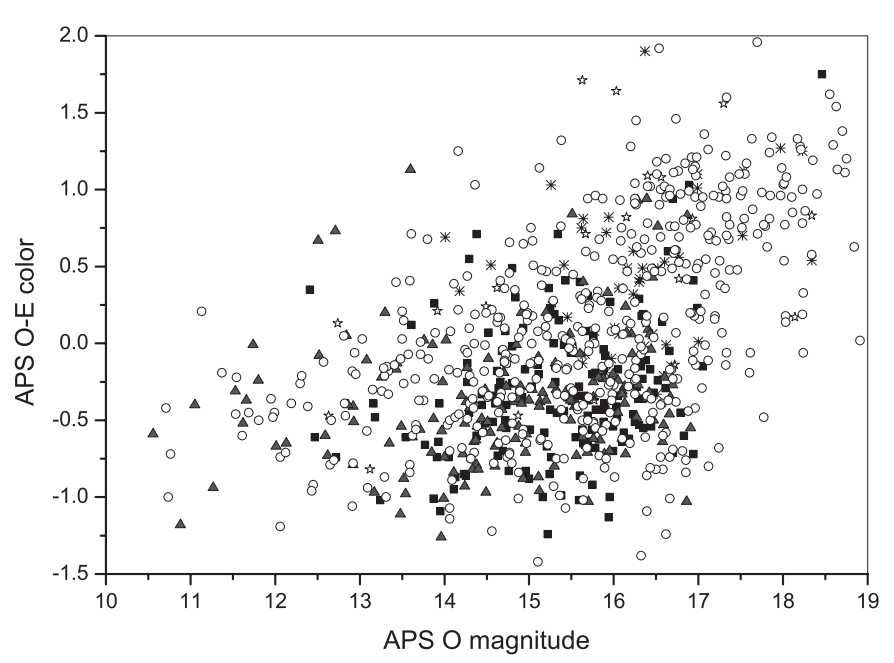

Fig. 9. MAPS color-magnitude diagram for 913 FBS BSOs. QSOs and Seyferts are indicated by asterisks, WDs by black squares, subdwarfs by black triangles, CVs by open stars, new (unknown) objects by open circles.

each found twice), the MAPS 4 "nodes" have been taken into account (in the MAPS, the artificial neural network classifier defines two nodes for each object, corresponding to the probability of being a star or a galaxy). Out of 913 objects, 879 are stars and 34 are galaxies (MAPS classes). However, out of these 34 "galaxies" 4 are known stars. During the position measurements we classified all FBS objects into star-like (including binaries, which are classified mostly as galaxies in MAPS), extended, and doubtful cases (to be checked later), and we adopt our classification for the future rather than the MAPS one.

There might be several reasons for non-coincidences: low $b$ (out of the MAPS area), variability (the object is absent in MAPS), or $>3^{\prime \prime}$ positional difference. Out of the 190 objects not found, 133 have $|b|<20^{\circ}$. 21 have $|b| \sim 20^{\circ}-22^{\circ}$, and may also be outside the area. Two are really strongly variable (see section Variable objects and binaries). But we are still left with 34 objects that should have been found in MAPS. We believe that our positions are better than $\sim 1^{\prime \prime}-1.5^{\prime \prime}$. On the other hand, MAPS positions are claimed to have $0.2^{\prime \prime} \mathrm{rms}$ error (maximum $1.5^{\prime \prime}$ near the plate edges). However, this is not always the case. Figure 8 shows the MAPS-FBS positional differences. We find objects at distances up to $3^{\prime \prime}$ (the MAPS-FBS rms difference is $0.89^{\prime \prime}$, and of course some objects may have larger positional differences).

The main purpose of the cross-correlation of FBS with MAPS was to have a homogeneous set of magnitudes and colors for the FBS objects. However, we plan to cross-correlate the FBS list with the USNO-B catalog too (Monet et al. 2003), to have these data for all objects down to lower $\mathrm{b}$ (a revised and updated catalog of the FBS blue stellar objects will be published separately). Figure 9 is the MAPS color-magnitude diagram for 913 FBS objects showing the color distribution of the FBS "blue" stellar objects (let us recall that FBS was aimed at detecting UV-excess stellar objects, UVX corresponding to $U-B$ rather than to $B-V$ or MAPS $O-E$; e.g. QSOs typically have $U-B<-0.5$ and $B-V>+0.5$ ). Known QSOs, WDs, subdwarfs, and CVs are shown with different symbols to 

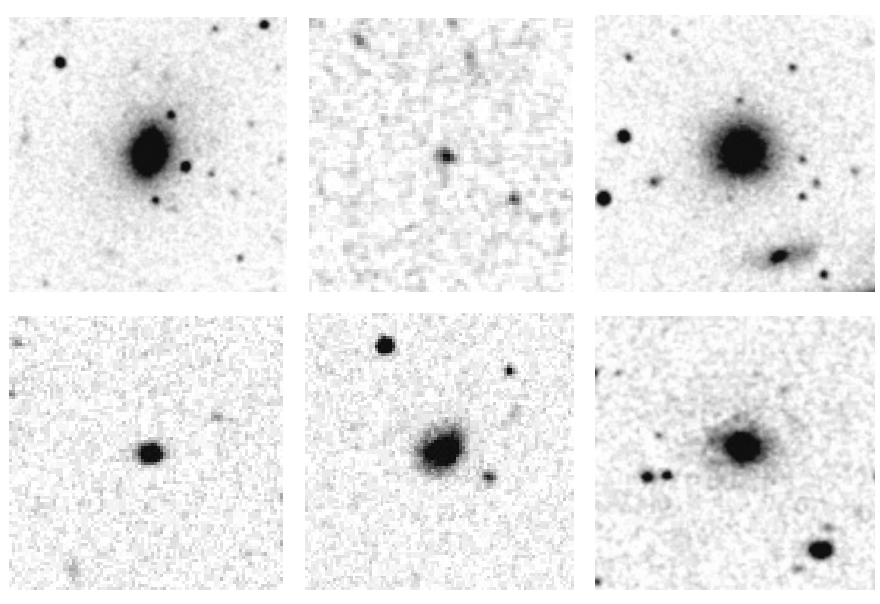

Fig. 10. Examples of compact galaxies among the FBS BSOs, candidate AGN.

distinguish them from objects of unknown nature. We see that a number of new good candidate QSOs and WDs may still be expected from the FBS.

\section{Research based on DSS1 and DSS2}

\subsection{AGN among the blue stellar objects}

During the position measurements we carefully inspected all three DSS images and, together with data from the FBS lowdispersion spectra, as well as radio, IR and X-ray data, we have made up a list of AGN candidates (QSOs and Seyferts). In all, FBS has re-discovered 30 known AGN (Véron-Cetty \& Véron 2003), and 12 FBS objects have been found to be new AGN (Mickaelian et al. 1999, 2001), the total number in the survey being 42 . However, only a small fraction of all objects has been observed spectroscopically, and therefore a number of new AGN can still be expected. On the other hand, as an exception, 8 extended objects have been included in the Second part of FBS, being good candidate Seyferts (only two are known; FBS 935 is MCG+07-35-050, and FBS 936 is MCG +07-35-061; but none of them is known as AGN or has been observed yet). In the MAPS catalog, 34 FBS objects are resolved as galaxies, including the 8 mentioned above, but only 3 of 42 known QSOs, 1 planetary nebula (PN, while we have found 3 more, having either extended images or images like binaries). 4 of the MAPS "galaxies" are known stars (WDs and subdwarfs). We have checked all DSS images for these 34 objects and find that only one of the images of the known stars shows signs of extension (an elongated image). There are 4 other binaries, 10 have pure stellar images (mostly established with the help of DSS2). Still, we are left with $15 \mathrm{ob}-$ jects, recognized as galaxies both by MAPS and our inspection (including the FBS 8 galaxies and 3 known QSOs, i.e. only 4 new objects). Note that some of the star-like images may turn out to be QSOs (but as the objects are bright we expect most of them to be stars, as bright QSOs should be resolved as extended). In all, from the DSS images we have found $73 \mathrm{QSO} /$ Seyfert candidates, but 22 of them are among the known QSOs and Seyferts (9 having a compact image with weak signs of extension, and 13 typical for galaxy images; thus 20 known QSOs out of 42 have pure stellar images).

21 are high-priority objects for spectroscopy. In Fig. 10 we give a few examples of DSS2r or DSS2b images (whichever is better) for new FBS AGN candidates.

\subsection{Proper motions}

Detectable proper motions (PM) are expected for nearby stars. Depending on their tangential velocity $\left(v_{\mathrm{t}}\right)$, the distance may be up to $\sim 1 \mathrm{kpc}$. Thus, PM can display either bright stars, or faint dwarf stars (WDs, subdwarfs, red dwarfs, and brown dwarfs). In the FBS sample there are no very bright stars, and the existence of red dwarfs and brown dwarfs is excluded (as the sample is for blue objects), so we are left with good candidate WDs and subdwarfs in case of detection of PM.

The differences between DSS2 and DSS1 for the FBS objects are up to $\sim 24^{\prime \prime}$. The accuracy of PM measurements depends on the separation of DSS1 and DSS2r epochs (3347 years in our sample). We consider that the DSS1 accuracy is $\sim 0.6^{\prime \prime}$, and the DSS2r is $\sim 0.4^{\prime \prime}$, according to our estimates. So the rms difference between the two positions is $\sim 0.8^{\prime \prime}$, which corresponds to an accuracy of PM measurements of $0.017-0.024^{\prime \prime} / \mathrm{yr}$.

Taking into account accidental errors in the measurements, the 869 objects having $<2^{\prime \prime}$ difference between the DSS1 and DSS2 positions may be excluded from the list of stars showing PM between these 2 epochs. We are left with 232 objects, which may have PM. However, to be secure, we have restricted the sample to 106 objects with $>3$ " differences $(3.75 \sigma$ limit) and we have checked the images and compared the configuration of stars in both DSS1 and DSS2. This was done with two methods: looking by eye for a change of stellar configuration (for the most obvious cases), and using new finding charts with exact centers of newly measured DSS1 positions with a great enlargement at the center of the images, and following a shift of position in the DSS2r image. During this examination, we have also marked the approximate direction (position angle) of the shift to compare later with the calculated one (from DSS2-DSS1) and increase the confidence or reject the object. Figure 11 shows three obvious cases of PM, where the shift in configuration can be detected even by eye (the upper image is DSS1, and the lower DSS2r). Out of 106 objects, we have detected PM for 78 objects.

It is worth mentioning that in 2 cases, FBS 197 and FBS 574, we were able to detect a PM even between DSS2r and DSS2b (positional differences 2.9" and 3.9", respectively). Thus, a careful study and comparison of DSS1 and DSS2 images may lead to the detection of PM stars.

Out of 78 objects with a high probability of PM detection, 57 are known from the Catalog of Spectroscopically Identified White Dwarfs (McCook \& Sion 2003; hereafter MCS), and only one is a subdwarf (PG). This confirms that from the FBS list objects having PM should turn to be WDs. So we have 20 new high-probability WD candidates. However, out of 58 known objects, PM was known for only 16, so we have detected PM for the first time for 42 additional objects. We have 


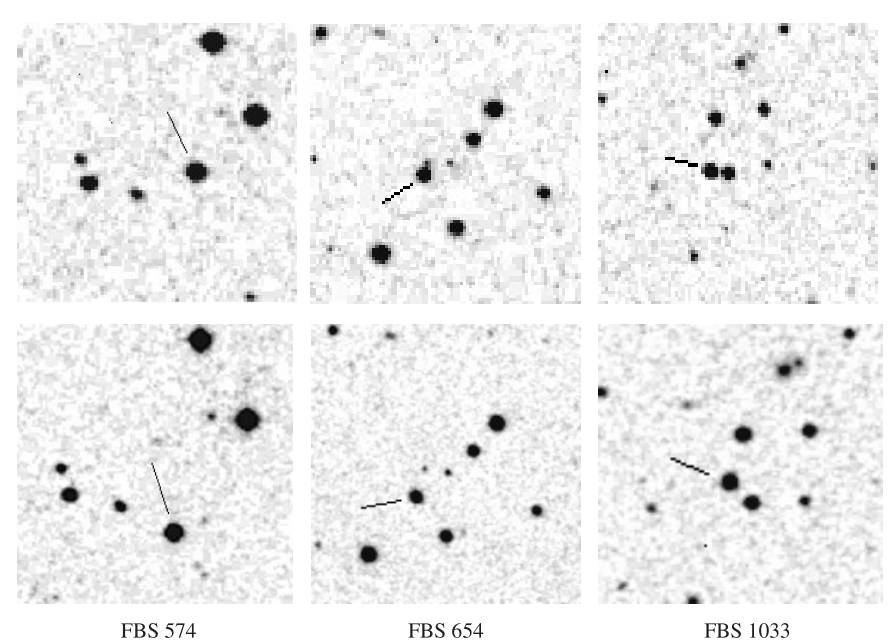

Fig. 11. Examples of obvious proper motion detected both by measurements and by inspection by eye of DSS1/DSS2 images. Upper images are DSS1 and lower are DSS2r for corresponding objects.

compared our data with those given in the USNO-B catalog as well (Monet et al. 2003). Table 2 is the list of these 78 objects with our measurements and those of USNO-B (for all objects) and previously known data from the MCS (for 16 objects). The consecutive columns list: 1 - FBS \#; 2 - FBS name (B1950 hhmm+ddd); 3 - magnitude; 4 - low-dispersion spectral type; 5 - DSS2r-DSS1 positional difference; 6 - PM found from our measurements; 7 - position angle of the motion; 8 - other names; 9 - types of objects; 10 - PM given in the MCS catalog; 11 - PM given in the USNO-B catalog. To check our detections, we have plotted in Fig. 12 the two measurements for the 16 objects with previously known PM. The agreement is within $0.079^{\prime \prime} / \mathrm{yr}$ with an $\mathrm{rms}$ of $0.041^{\prime \prime} / \mathrm{yr}$ (errors of PM are not given in MCS, but most probably, in view of the separation in years, our measurements are better). The rms in the difference of our measurements and those of USNO-B is $0.106^{\prime \prime} / \mathrm{yr}$ due to larger differences because of a few objects with 0 PM in USNO-B.

In the MCS catalog, distances are given for 289 objects, and PM are known for 1293 objects. 272 objects have both measured PM and distances, the tangential velocities being typically within $100 \mathrm{~km} \mathrm{~s}^{-1}$. Accepting this value as a typical upper limit for $v_{\mathrm{t}}$, we find that an object having $\mathrm{PM}=0.1^{\prime \prime} / \mathrm{yr}$ (the typical value for our new 20 objects), should be at a distance of less than $210 \mathrm{pc}$ and for $m=15$ have $M_{\mathrm{abs}}$ fainter than $8.4^{\mathrm{m}}$. Except for the very bright object FBS 660 with MAPS O = 11.61, and FBS 613 (FBS $m=13.7$ ), all other 18 objects have MAPS magnitudes fainter than $15^{\mathrm{m}}$, and the calculated upper limits for their $M_{\mathrm{abs}}$ when $v_{\mathrm{t}}=100 \mathrm{~km} \mathrm{~s}^{-1}$ are $9^{\mathrm{m}}$ and fainter. Given that the objects are blue, this makes them either WDs or subdwarfs. However, the $v_{\mathrm{t}}$ limit we take is larger than the typical values for most of the objects $\left(v_{\mathrm{t}} \sim 50 \mathrm{~km} \mathrm{~s}^{-1}\right)$, and many of these objects are WD candidates from their FBS lowdispersion spectra too. So we consider these objects as highly probable WDs.

It is worth mentioning that among the other 28 FBS objects with $>3^{\prime \prime}$ differences between DSS2r and DSS1, we find in the USNO-B catalog only 3 objects with $\mathrm{PM}>0.068^{\prime \prime} / \mathrm{yr}$ (the smallest value in our list of 78), most of them having 0 or negligible PM. So our two methods to check the reality of the PM helped to find and eliminate false differences between the positions of DSS1 and DSS2.

Finally, we find that among 42 known FBS QSOs, 33 have DSS2r-DSS1 differences within $1^{\prime \prime}$ and only 4 have $>2^{\prime \prime}$ (the largest being $2.7^{\prime \prime}$ ), with an rms of $0.98^{\prime \prime}$. For the known FBS WDs (altogether 88 out of a total of 145 , excluding those having DSS2r-DSS1 > 3"), only $27(31 \%$ compared to $79 \%$ for QSOs) have DSS2r-DSS1 < 1", and the rms difference is $1.80^{\prime \prime}$. This difference between DSS2r and DSS1 for QSOs and WDs shows that differences $<3$ " could also be explained partly by PM. Thus, the objects having DSS2r-DSS1 $>2^{\prime \prime}$ are also good candidates for having PM and being WDs and subdwarfs. Among the 126 objects with $2^{\prime \prime}<$ DSS2r-DSS $23^{\prime \prime}$, and 28 with $>3$ ", but no visually detected PM, 25 are known WDs, 26 are subdwarfs, 3 are CVs, and 4 are QSOs (the fraction of subdwarfs becomes significant). We are left with 96 new objects. But because of accidental errors in measurements, we cannot refer all these differences to PM.

\subsection{Variable objects and binaries}

During the position measurements, we also paid attention to brightness differences of objects and binary images, and we compare the DSS data to those from the FBS plates. This (together with the low-dispersion spectral data from FBS, namely emission features in case of $\mathrm{CVs}$ ) reveals candidate $\mathrm{CVs}$ for further spectroscopic observations. Figure 13 shows a significant change in brightness from DSS1 to DSS2 for 2 objects: FBS 218 has become brighter, and FBS 882 fainter. FBS 882 has MAPS O $=18.14$ (DSS1 epoch), but in DSS2r it is near the plate limit and should be $\sim 21^{\mathrm{m}}$. FBS 218 has no MAPS detection, but its FBS magnitude is $15.5^{\mathrm{m}}$, which is much brighter than DSS1 (near the plate limit, $\sim 21^{\mathrm{m}}$ ), as well as the estimated DSS2r magnitude $\left(\sim 18^{\mathrm{m}}\right)$.

In a number of cases, the MAPS magnitudes (compared with the FBS ones) helped us to find large differences in brightness $\left(16\right.$ objects with MAPS-FBS $>2.5^{\mathrm{m}}$ compared to $\mathrm{rms}$ of $\left.0.85^{\mathrm{m}}\right)$, though we have concluded for variability only after additionally checking the images in comparison with the neighboring stars (to avoid errors in our approximate estimation of magnitudes from the FBS plates, especially for objects of lists 1-4). All these 16 objects are good CV candidates.

There are 69 close binary images among the FBS objects with a separation of $3^{\prime \prime}-10^{\prime \prime}$ detected in all three: DSS1, DSS2r and DSS2b (and $\sim 100$ more seen in 1 or 2 images); this is not enough for classification as a physical binary or $\mathrm{CV}$, but can support other criteria if available. Out of 33 classified objects, 14 are WDs, 17 are subdwarfs, and 2 are CVs. Others are candidates for spectroscopic observations. Most of them might be physical binaries, as among QSOs there are no such cases, proving that chance superposition of images is rare at high galactic latitudes.

A subsample of candidate CVs has been compiled both on the basis of the FBS spectroscopic data and using the signs 
Table 2. The list of 78 FBS objects with detected PM between DSS2 and DSS1.

\begin{tabular}{|c|c|c|c|c|c|c|c|c|c|c|}
\hline FBS \# & FBS name & $m$ & LDS & $\begin{array}{r}\text { DSS2r-DSS1 } \\
\end{array}$ & $\begin{array}{l}\mathrm{PM} \\
\text { "/yr }\end{array}$ & $\begin{array}{r}\mathrm{PA} \\
\circ\end{array}$ & Other names & Type & $\begin{array}{l}\text { MCS } \\
\prime \prime / y r\end{array}$ & $\begin{array}{c}\text { USNO-B } \\
" 1 / \mathrm{yr}\end{array}$ \\
\hline 430 & $0004+330$ & 14.1 & $\mathrm{~B} 1$ & 3.0 & 0.078 & 231 & GD 002, EG203 & DA1 & & 0.099 \\
\hline 443 & $0050+358$ & 14.6 & B1 & 4.6 & 0.121 & 190 & RE, EUV & DA2 & & 0.116 \\
\hline 449 & $0106+353$ & 15.0 & $\mathrm{~B} 2$ & 9.5 & 0.239 & 337 & & & & 0.219 \\
\hline 9 & $0106+372$ & 14.0 & B2 & 6.4 & 0.160 & 111 & GD 011, EG179 & DA1.5 & & \\
\hline 20 & $0150+396$ & 16.0 & B2a: & 4.4 & 0.122 & 132 & & & & 0.000 \\
\hline 460 & $0213+355$ & 15.5 & B2 & 3.8 & 0.109 & 205 & & & & 0.100 \\
\hline 32 & $0259+378$ & 15.0 & $\mathrm{~B} 2$ & 4.2 & 0.119 & 116 & GD 038, GR287 & DA3 & 0.191 & 0.121 \\
\hline 470 & $0316+345$ & 14.8 & B1a & 7.2 & 0.191 & 170 & EG208, GD045 & DA3.5 & 0.155 & 0,192 \\
\hline 238 & $0625+415$ & 14.0 & $\mathrm{~B} 2$ & 5.1 & 0.151 & 167 & GD 074 & DA3 & & 0.111 \\
\hline 654 & $0712+623$ & 15.9 & $\mathrm{~B} 2$ & 6.3 & 0.143 & 173 & & & & 0.205 \\
\hline 44 & $0716+404$ & 15.5 & B1 & 6.9 & 0.188 & 125 & GD 085, EG216 & DB3 & & 0.171 \\
\hline 660 & $0742+625$ & 11.9 & B1 & 4.1 & 0.094 & 8 & & & & 0.004 \\
\hline 246 & $0743+442$ & 13.5 & N1e: & 6.0 & 0.169 & 209 & GR343, GD089 & DA5 & & 0.182 \\
\hline 663 & $0811+645$ & 14.6 & B1a & 8.1 & 0.184 & 261 & GD 457 & DA4 & & 0.193 \\
\hline 53 & $0817+386$ & 16.0 & $\mathrm{~N} 2$ & 4.6 & 0.123 & 265 & KUV, PG & DA2 & & 0.105 \\
\hline 872 & $0817+721$ & 15.5 & $\mathrm{~B} 2 \mathrm{a}$ & 6.9 & 0.163 & 191 & & & & 0.158 \\
\hline 253 & $0826+455$ & 15.5 & B3a & 6.1 & 0.170 & 220 & LTT12215, EG217, GD 91, PG & DA5 & 0.210 & 0.000 \\
\hline 58 & $0854+405$ & 14.5 & N1a & 7.3 & 0.198 & 175 & GD 098, PG & DA2.5 & 0.180 & 0.190 \\
\hline 491 & $0858+363$ & 15.2 & $\mathrm{~N} 2$ & 7.7 & 0.227 & 204 & GD 099, PG & DAV4 & & 0.196 \\
\hline 496 & $0920+364$ & 16.4 & $\mathrm{~N} 2$ & 4.3 & 0.096 & 159 & TON1054, PG & DA4 & & 0.089 \\
\hline 266 & $0941+432$ & 16.4 & $\mathrm{~B} 1$ & 3.4 & 0.081 & 195 & US 906, PG & DA4 & & 0.084 \\
\hline 268 & $0943+441$ & 14.1 & B1a & 12.8 & 0.304 & 4 & G116-052, EGGR 67, PG & DA4 & 0.297 & 0.290 \\
\hline 272 & $1002+430$ & 15.0 & B1 & 5.2 & 0.141 & 247 & GD 111 & DA3 & & 0.301 \\
\hline 680 & $1005+642$ & 14.2 & $\mathrm{~B} 1$ & 5.7 & 0.129 & 230 & GD 462, PG & DA2 & & 0.131 \\
\hline 73 & $1032+406$ & 10.8 & $\mathrm{~B} 1$ & 4.3 & 0.117 & 247 & PG & $\mathrm{sdB}$ & & 0.094 \\
\hline 281 & $1039+412$ & 16.0 & $\mathrm{~B} 2 \mathrm{a}$ & 3.3 & 0.073 & 78 & PB 520, PG & DA & & 0.075 \\
\hline 987 & $1039+748$ & 15.2 & B2a: & 3.4 & 0.076 & 191 & PG & DA1.5 & & 0.067 \\
\hline 885 & $1048+715$ & 15.7 & $\mathrm{~B} 3 \mathrm{a}$ & 6.4 & 0.140 & 224 & & & & 0.158 \\
\hline 508 & $1056+345$ & 15.8 & $\mathrm{~N} 1$ & 13.5 & 0.301 & 219 & G119-047, PG & DB5 & 0.281 & 0.285 \\
\hline 991 & $1102+749$ & 14.8 & B1a & 5.3 & 0.120 & 251 & GD 466, PG & DA2.5 & & 0.120 \\
\hline 688 & $1103+619$ & 15.9 & B3 & 4.4 & 0.116 & 170 & & & & 0.062 \\
\hline 81 & $1119+386$ & 16.0 & B1a: & 3.9 & 0.108 & 275 & PG & DA4 & & 0.088 \\
\hline 287 & $1120+439$ & 15.3 & B1a & 3.9 & 0.090 & 281 & PG, RE & DA1 & & 0.060 \\
\hline 82 & $1129+373$ & 16.2 & B1 & 4.3 & 0.094 & 95 & PG & DB4 & & 0.095 \\
\hline 800 & $1136+668$ & 13.3 & $\mathrm{~B} 1$ & 3.5 & 0.083 & 252 & HS & DAO & & 0.065 \\
\hline 88 & $1211+393$ & 17.0 & $\mathrm{~B} 2$ & 3.9 & 0.116 & 290 & LP216-074, EG255, CBS & DA3 & 0.132 & 0.136 \\
\hline 307 & $1230+417$ & 15.7 & $\mathrm{~B} 1$ & 3.8 & 0.095 & 274 & $\mathrm{HZ} 28, \mathrm{GD} 317, \mathrm{PG}$ & DA2 & & 0.102 \\
\hline 811 & $1231+680$ & 17.4 & $\mathrm{~N} 2$ & 5.0 & 0.110 & 291 & & & & 0.105 \\
\hline 1003 & $1240+754$ & 15.4 & $\mathrm{~B} 2 \mathrm{a}$ & 7.3 & 0.174 & 263 & LB 261, PG & DA4 & 0.230 & 0.190 \\
\hline 706 & $1241+651$ & 16.0 & B2 & 5.3 & 0.133 & 216 & GD 479, GR292 & DB4 & & 0.153 \\
\hline 531 & $1254+345$ & 17.0 & $\mathrm{~N} 2$ & 3.5 & 0.089 & 145 & HS & DAH & & 0.040 \\
\hline 317 & $1255+426$ & 16.2 & $\mathrm{~B} 1$ & 3.2 & 0.068 & 228 & PG & DA3 & & 0.074 \\
\hline 535 & $1307+354$ & 15.7 & $\mathrm{~B} 2$ & 7.1 & 0.182 & 266 & GR537, BG CVn, PG & DA5 & & 0.228 \\
\hline 711 & $1322+627$ & 15.8 & $\mathrm{~B} 2$ & 3.5 & 0.078 & 167 & & & & 0.045 \\
\hline 897 & $1337+705$ & 12.8 & $\mathrm{~B} 1$ & 15.0 & 0.393 & 261 & LTT18341, PG & DA2 & 0.380 & 0.402 \\
\hline 1012 & $1344+765$ & 16.3 & $\mathrm{~B} 2$ & 4.2 & 0.101 & 240 & & & & 0.589 \\
\hline 825 & $1350+657$ & 15.4 & $\mathrm{~B} 2 \mathrm{a}$ & 9.6 & 0.276 & 301 & LP066-262, GR538, PG & DA5 & 0.280 & 0.284 \\
\hline 109 & $1403+386$ & 17.0 & $\mathrm{~B} 2$ & 4.7 & 0.118 & 170 & & & & 0.104 \\
\hline 1015 & $1405+749$ & 15.5 & B1a & 3.6 & 0.088 & 220 & & & & 0.143 \\
\hline 334 & $1410+425$ & 16.5 & B2 & 4.0 & 0.098 & 224 & PB 1665, PG & DA4 & & 0.097 \\
\hline 338 & $1422+433$ & 17.0 & N1 & 4.5 & 0.097 & 308 & CBS183 & DA & & 0.085 \\
\hline 113 & $1428+374$ & 14.9 & $\mathrm{~B} 2 \mathrm{a}$ & 4.5 & 0.107 & 165 & KUV, PG & DA & & 0.086 \\
\hline 115 & $1429+373$ & 15.4 & B1a: & 4.1 & 0.098 & 271 & EG236, KUV, PG & DA1 & & 0.111 \\
\hline 1019 & $1440+753$ & 15.2 & B1 & 3.5 & 0.081 & 272 & RE, EUV & DA1 & & 0.076 \\
\hline 1086 & $1459+821$ & 14.9 & B1e: & 12.8 & 0.327 & 301 & G256-018, LP008-157 & DB4 & 0.330 & 0.364 \\
\hline 558 & $1502+351$ & 15.9 & B2 & 4.6 & 0.120 & 309 & PG & DA4 & & 0.117 \\
\hline
\end{tabular}


Table 2. continued.

\begin{tabular}{|c|c|c|c|c|c|c|c|c|c|c|}
\hline FBS \# & FBS name & $m$ & LDS & $\begin{array}{r}\text { DSS2r-DSS1 } \\
\prime \prime\end{array}$ & $\begin{array}{l}\mathrm{PM} \\
\prime / \mathrm{yr}\end{array}$ & $\begin{array}{r}\mathrm{PA} \\
\circ\end{array}$ & Association & Type & $\begin{array}{r}\text { MCS } \\
" / \mathrm{yr}\end{array}$ & $\begin{array}{c}\text { USNO-B } \\
" / \mathrm{yr}\end{array}$ \\
\hline 724 & $1508+637$ & 15.2 & B1a & 4.7 & 0.123 & 205 & GR364, PG & DA5 & & 0.129 \\
\hline 727 & $1518+637$ & 16.3 & B2a & 8.4 & 0.215 & 306 & LP068-060, G224-059 & DA3 & 0.250 & 0.233 \\
\hline 574 & $1559+369$ & 14.2 & N1 & 23.8 & 0.540 & 168 & LHS3146, G180-023, PG & DAV5 & 0.570 & 0.292 \\
\hline 365 & $1608+419$ & 16.5 & B2 & 4.6 & 0.129 & 160 & PG & DA4 & & 0.000 \\
\hline 1033 & $1627+747$ & 15.8 & $\mathrm{~B} 2 \mathrm{a}$ & 8.6 & 0.208 & 1 & HS & DA3.6 & & 0.173 \\
\hline 577 & $1637+335$ & 15.2 & B2 & 19.1 & 0.491 & 186 & L1491-027, KUV, PG & DA5 & 0.570 & 0.347 \\
\hline 142 & $1641+388$ & 14.9 & $\mathrm{~N} 3 \mathrm{a}$ & 5.1 & 0.127 & 22 & BPM91679, EG238, PG & DA6 & & 0.171 \\
\hline 746 & $1645+649$ & 15.9 & B1a: & 3.4 & 0.089 & 181 & & & & 0.078 \\
\hline 748 & $1654+637$ & 15.6 & B3a & 7.5 & 0.186 & 231 & GD 515, PG & DA4 & & 0.150 \\
\hline 376 & $1658+440$ & 15.0 & B1 & 3.9 & 0.106 & 346 & PG, RE & DAP2 & & 0.106 \\
\hline 596 & $1713+333$ & 15.4 & B2 & 7.6 & 0.196 & 148 & ВPM92172, GD 360, PG & DA2 & & 0.345 \\
\hline 912 & $1713+696$ & 13.1 & B1a & 15.9 & 0.395 & 181 & $\mathrm{GW}+696829, \mathrm{G} 240-051$ & DA3 & 0.340 & 0.170 \\
\hline 383 & $1737+419$ & 15.0 & B1a: & 6.9 & 0.176 & 355 & GD 363, EG240 & DA3 & & 0.188 \\
\hline 157 & $1743+403$ & 17.0 & $\mathrm{~B} 2$ & 3.2 & 0.082 & 151 & & & & 0.093 \\
\hline 158 & $1749+393$ & 15.5 & B1a: & 3.1 & 0.078 & 220 & & & & 0.000 \\
\hline 855 & $1800+686$ & 15.5 & $\mathrm{~B} 1$ & 3.4 & 0.083 & 206 & RE, KUV & DA1 & & 0.095 \\
\hline 170 & $1822+410$ & 15.5 & B1 & 4.1 & 0.111 & 357 & EG242, GD 378 & DBA3 & & 0.138 \\
\hline 613 & $2154+329$ & 13.7 & B1 & 3.4 & 0.090 & 87 & & & & 0.035 \\
\hline 620 & $2212+335$ & 15.3 & B1 & 5.3 & 0.159 & 146 & & & & 0.000 \\
\hline 189 & $2254+373$ & 17.0 & $\mathrm{~B} 2 \mathrm{a}$ & 5.1 & 0.148 & 119 & & & & 0.147 \\
\hline 197 & $2329+407$ & 13.0 & B1a & 12.6 & 0.340 & 118 & EG160, G171-002 & DA3 & 0.280 & 0.280 \\
\hline 203 & $2354+375$ & 16.0 & B1 & 3.2 & 0.080 & 150 & & & & 0.062 \\
\hline
\end{tabular}

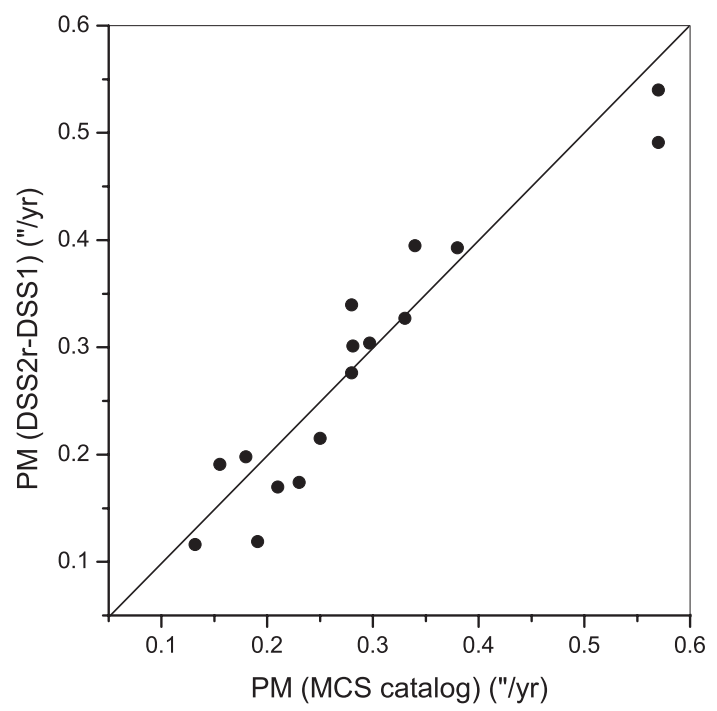

Fig. 12. Plot of PM detected by DSS 1 and DSS 2 measurements vs. PM given in MCS catalog for 16 FBS BSOs. A $45^{\circ}$ line is given to help the eye and is not a fit.

from inspection of DSS images mentioned above. FBS has rediscovered 21 known CVs from the Catalogue of cataclysmic variables, low-mass X-ray binaries and related objects (Ritter \& Kolb 2003), and 7 other binaries. We estimate that in total there are $\sim 35$ more new CVs in the FBS.

We have selected two emission-line objects (FBS 1096 and FBS 1098), which are absent in both DSS1 and DSS2 (red and blue) charts. One has $\sim 13.5^{\mathrm{m}} B$ magnitude on the FBS plate, and the other $\sim 14^{\mathrm{m}}$. The DSS1 blue plate of that area (POSS \#802 for both objects) has a detection limit of $21.61^{\mathrm{m}}$

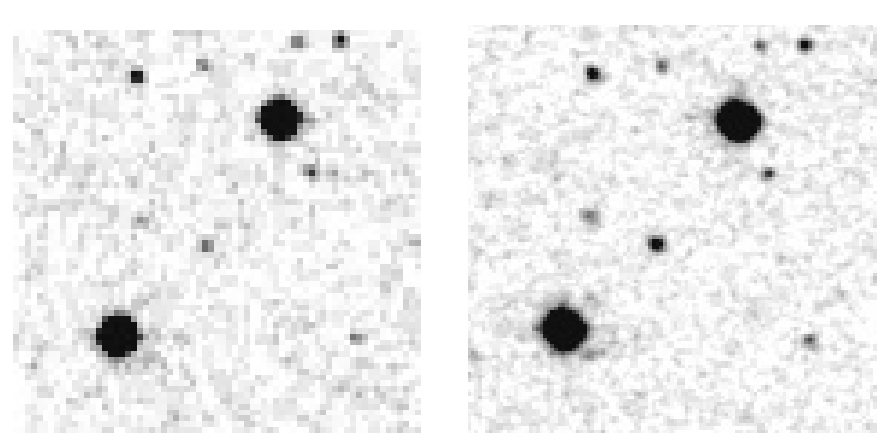

FBS 218

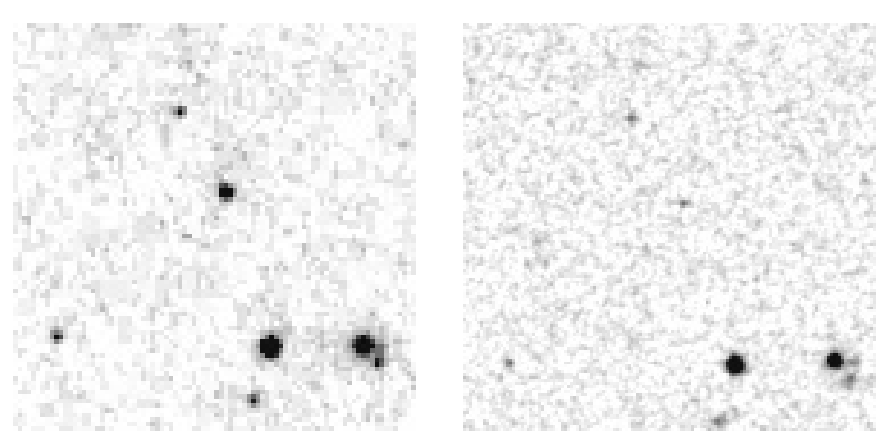

FBS 882

Fig. 13. Examples of variable FBS BSOs detected by comparison of DSS1 and DSS2r images. Both objects are the nearest to the center.

according to MAPS (DSS2b being even deeper, but data for its limit are not available), so the estimated lower limit for the amplitude is $8^{\mathrm{m}}$ and $7.5^{\mathrm{m}}$, respectively. We believe these are 
probably Novae, and plan to obtain deep images in these fields to detect the progenitor stars.

\section{Conclusion}

We have carried out accurate position measurements for 153 AGN having absolute VLBI positions, and for 1101 FBS blue stellar objects. A number of errors in both DSS1 and DSS2 has been found; if only measuring on a single image, some accidental errors may occur. For the first time, the DSS2 astrometric accuracy and DSS1-DSS2 positional agreement have been established. The rms errors are: 0.64 " for DSS1, $0.46^{\prime \prime}$ for DSS2 red, and $0.83^{\prime \prime}$ for DSS2 blue, and the maximum available accuracy of $0.42^{\prime \prime}$ is obtained by weighted averaging between DSS1 and DSS2 red. New accurate positions for 1101 FBS objects are given, making a dramatic improvement in their positions which sometimes had errors of a few arcmin. The accuracy of the FBS lists has been established for lists 1-4 (59.'2) and for 5-11 (14.'9), as well as for PG (16.'8). A number of misprints and wrong FBS and PG positions have been corrected. PM for $78 \mathrm{WD}$ candidates have been measured, including 62 for the first time. Candidate QSO/Sy, WDs and CVs have been found. Cross-correlation of the FBS list with the MAPS catalog has been made, establishing homogeneous magnitudes and colors for 913 FBS objects.

Acknowledgements. The author is grateful to P. Véron and M.-P. Véron-Cetty (Observatoire de Haute-Provence) for useful discussions and a number of comments on the draft of the paper, and to R.C. Stone (USNO) for useful comments for the improvement of the paper.

The Digitized Sky Surveys were produced at the Space Telescope Science Institute under US Government grant NAG W-2166. The images of these surveys are based on photographic data obtained using the Oschin Schmidt Telescope on Palomar Mountain and the UK Schmidt Telescope. The plates were processed into the present compressed digital form with the permission of these institutions. The National Geographic Society - Palomar Observatory Sky Atlas (POSS-I) was made by the California Institute of Technology with grants from the National Geographic Society.

The Second Palomar Observatory Sky Survey (POSS-II) was made by the California Institute of Technology with funds from the National Science Foundation, the National Geographic Society, the Sloan Foundation, the Samuel Oschin Foundation, and the Eastman Kodak Corporation. The Oschin Schmidt Telescope is operated by the California Institute of Technology and Palomar Observatory.

The UK Schmidt Telescope was operated by the Royal Observatory Edinburgh, with funding from the UK Science and Engineering Research Council (later the UK Particle Physics and Astronomy Research Council), until 1988 June, and thereafter by the AngloAustralian Observatory. The blue plates of the southern Sky Atlas and its Equatorial Extension (together known as the SERC-J), as well as the Equatorial Red (ER), and the Second Epoch [red] Survey (SES) were all taken with the UK Schmidt.

\section{References}

Abrahamian, H. V., \& Mickaelian, A. M. 1996, Ap, 39, 315

Abrahamian, H. V., Lipovetsky, V. A., Mickaelian, A. M., \& Stepanian, J. A. 1999, CDS, Catalog No. II/223

Bicay, M. D., Stepanian, J. A., Chavushyan, V. H., et al. 2000, A\&AS, 147,169

Bowen, D. V., Osmer, S. J., Blades, J. C., et al. 1994, AJ 107, 461

Cabanela, J. E., Humphreys, R. M., Aldering, G., et al. 2003, PASP, 115,837

Cotton, B. 1999, http://www.cv.nrao.edu/bcotton/ fitsview.html

Green, R. F., Schmidt, M., \& Liebert, J. 1986, ApJS, 61, 305

Heckman, O., Dickevoss, W., Kox, H., Grunter, A., \& Brosterhus, E. 1975, AGK 3, Hamburg-Bergedorf

Hog, E., Fabricius, C., Makarov, V. V., et al. 2000, The Tycho-2 Catalogue of the 2.5 Million Brightest Stars, A\&A, 355, L27

Kirhakos, S., Sargent, W. L. W., Schneider, D. P., et al. 1994, PASP, 106,646

Lasker, B. M., Sturch, C. R., McLean, B. J., et al. 1990, AJ, 99, 2019

Lasker, B. M., Doggett, J., McLean, B., et al. 1996, ASP Conf. Ser., 88

Lasker, B. M., Greene, G. R., Lattanzi, M. G., McLean, B. J., \& Volpicelli, A. 1998, Proc. Workshop Astrophysics and Algorithms

Ma, C., Shaffer, D. B., de Vegt, C., Johnston, K. J., \& Russell, J. L. 1990, AJ, 99, 1284

Markarian, B. E. 1967, Astrofizika, 3, 55

Markarian, B. E., Lipovetski, V. A., Stepanian, J. A., Erastova, L. K., \& Shapovalova, A. I. 1989, Commun. Special Astrophys. Obs., 62,5

Mazzarella, J., \& Balzano, V. 1986, ApJS, 62, 751

McCook, G. P., \& Sion, E. M. 1999, ApJS, 121, 1 (Version June 2003 in CDS, Catalog No. III/235)

McLean, B. J. 2000, ADASS X meeting, Boston, USA

Meisenheimer, K., \& Roser, H. J. 1983, A\&AS, 51, 41

Mickaelian, A. M. 2000, Astron. Astrophys. Transactions, 18, 557

Mickaelian, A. M., Gonçalves, A. C., Véron-Cetty, M. P., \& Véron, P. 1999, Ap, 42, 1

Mickaelian, A. M., Gonçalves, A. C., Véron-Cetty, M. P., \& Véron, P. 2001, Ap, 44, 14

Mickaelian, A. M., Balayan, S. K., Ilovaisky, S. A., et al. 2002, A\&A, 381, 894

Monet, D. G., Levine, S. E., Casian, B., et al. 2003, AJ, 125, 984

Ritter, H., \& Kolb, U. 2003, A\&A, 404, 301

Robertson, D. S., Carter, W. E., Ray, J. R., et al. 1993, AJ, 105, 353

Russell, J. L., Lasker, B. M., McLean, B. J., et al. 1990, AJ, 99, 2059

SAO 1966, Smithsonian Astrophysical Observatory Star Catalog, Smithsonian Institution, Washington, DC

Schmidt, M., \& Green, R. F. 1983, ApJ, 269, 352

Schneider, D. P., Bahcall, J. N., Saxe, D. H., et al. 1992, PASP, 104, 678

Sovers, O. J., Edwards, C. D., Jacobs, C. S., et al. 1988, AJ, 95, 1647

Stickel, M., \& Kuehr, H. 1994, A\&AS, 103, 349

Stoy, R. H. 1968, Ann. Cape Obs. XXII

Véron, P., \& Hawkins, M. R. S. 1995, A\&A, 296, 665

Véron-Cetty, M.-P., \& Véron, P. 1996, A\&AS, 115, 97

Véron-Cetty, M.-P., \& Véron, P. 2003, A\&A, 412, 399 